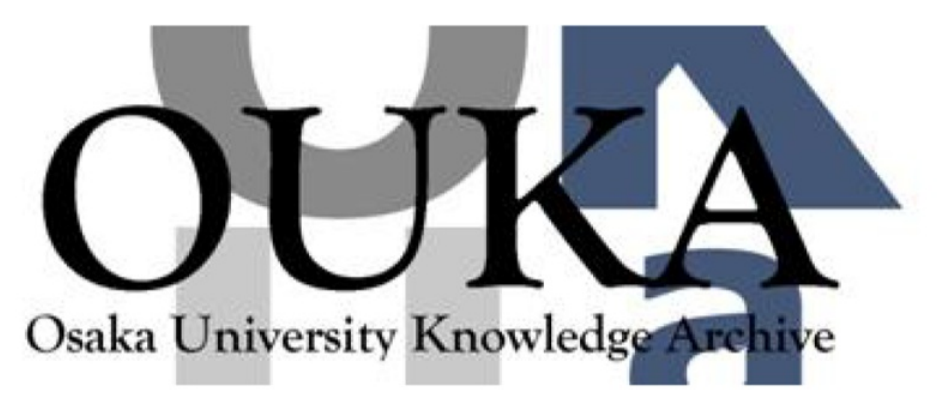

\begin{tabular}{|c|c|}
\hline Title & $\begin{array}{l}\text { Effect of spin-orbit impurity scattering on the } \\
\text { Knight shift in BCS superconductors: } \\
\text { Reanalysis of Anderson's classic paper }\end{array}$ \\
\hline Author (s) & Miyake, K.; Asayama, K. \\
\hline Citation & Physical Review B. 62(17) p.11363-p. 11364 \\
\hline Issue Date & $2000-11-01$ \\
\hline oaire:version & VoR \\
\hline URL & https://hdl. handle. net/11094/3234 \\
\hline rights & $\begin{array}{l}\text { Miyake, K., Asayama, K., Physical Review B, } 62 \text {, } \\
17,11363-1364,2000-11-01 \text {. "Copyright }(2000) \\
\text { by the American Physical Society." }\end{array}$ \\
\hline Note & \\
\hline
\end{tabular}

Osaka University Knowledge Archive : OUKA

https://ir. Library. osaka-u. ac. jp/

Osaka University 


\title{
Effect of spin-orbit impurity scattering on the Knight shift in BCS superconductors: Reanalysis of Anderson's classic paper
}

\author{
K. Miyake and K. Asayama \\ Division of Materials Physics, Department of Physical Science, Graduate School of Engineering Science, Osaka University, \\ Toyonaka, Osaka 560-8531, Japan \\ (Received 16 June 2000)
}

\begin{abstract}
We present the result of a numerical reanalysis of the classic paper of Anderson discussing the effect of spin-orbit scattering on the Knight shift in BCS conventional superconductors. The numerical evaluation of Anderson's relation between the Knight shift and the scattering rates improves on the approximate numerical factors given by Anderson.
\end{abstract}

The aim of this Brief Report is very simple. We have reanalyzed numerically the formula for the effect of impurity scattering with spin-orbit coupling on the Knight shift given by Anderson in the classic paper of this field, ${ }^{1}$ and realized that the numerical calculations performed about four decades ago might be replaced by an updated version.

Skipping the derivations, we present the formula for the spin susceptibility given by Eq. (8) in Ref. 1:

$$
\frac{\chi_{\mathrm{s}}}{\chi_{\mathrm{n}}}=\frac{\int d \epsilon \int d \epsilon^{\prime} f\left(\left(\epsilon-\epsilon^{\prime}\right) \tau \hbar^{-1}\right)\left[u(\epsilon) v\left(\epsilon^{\prime}\right)-v(\epsilon) u\left(\epsilon^{\prime}\right)\right]^{2}\left(E+E^{\prime}\right)^{-1}}{\int d \epsilon \int d \epsilon^{\prime} \lim _{\Delta \rightarrow 0} f\left(\left(\epsilon-\epsilon^{\prime}\right) \tau \hbar^{-1}\right)\left[u(\epsilon) v\left(\epsilon^{\prime}\right)-v(\epsilon) u\left(\epsilon^{\prime}\right)\right]^{2}\left(E+E^{\prime}\right)^{-1}},
$$

where $\tau$ is the lifetime of the quasiparticle due to spin-orbit scattering from impurities or the boundary of the system, the coefficients $u$ and $v$ are defined as

$$
\begin{aligned}
& u(\epsilon)=\frac{1}{\sqrt{2}}\left(1+\frac{\epsilon}{E}\right)^{1 / 2}, \\
& v(\epsilon)=\frac{1}{\sqrt{2}}\left(1-\frac{\epsilon}{E}\right)^{1 / 2},
\end{aligned}
$$

where $\epsilon$ is the energy level of a one-particle state in the normal state measured from the chemical potential, and the corresponding one-particle excitation energies $E$ and $E^{\prime}$ in the superconducting state are defined in terms of the superconducting gap $\Delta$ as usual:

$$
E=\sqrt{\epsilon^{2}+\Delta^{2}} \text { and } E^{\prime}=\sqrt{\epsilon^{\prime 2}+\Delta^{2}} .
$$

The relaxation function $f$ in Eq. (1) is assumed to be given by the Lorentzian form

$$
f(x)=\frac{1}{1+x^{2}}
$$

In the above expressions, $\epsilon_{0}$ in Ref. 1 has been replaced by $\Delta$, a much more familiar notation now.

Using the definitions (2), (3), and (4), the coherence factor $\left[u(\epsilon) v\left(\epsilon^{\prime}\right)-v(\epsilon) u\left(\epsilon^{\prime}\right)\right]^{2}$ is calculated in the standard way:

$$
\left[u(\epsilon) v\left(\epsilon^{\prime}\right)-v(\epsilon) u\left(\epsilon^{\prime}\right)\right]^{2}=\frac{E E^{\prime}-\epsilon \epsilon^{\prime}-\Delta^{2}}{E E^{\prime}} .
$$

Then, the expression for $\chi_{\mathrm{s}} / \chi_{\mathrm{n}}$ is given in the following form:

$$
\frac{\chi_{\mathrm{s}}}{\chi_{\mathrm{n}}}=\frac{1}{2 \pi} \int_{-\infty}^{+\infty} d x \int_{-\infty}^{+\infty} d x^{\prime} \frac{1}{1+\left(x-x^{\prime}\right)^{2}} \frac{\sqrt{x^{2}+\left(\Delta \tau \hbar^{-1}\right)^{2}} \sqrt{x^{\prime 2}+\left(\Delta \tau \hbar^{-1}\right)^{2}}-x x^{\prime}-\left(\Delta \tau \hbar^{-1}\right)^{2}}{\sqrt{x^{2}+\left(\Delta \tau \hbar^{-1}\right)^{2}} \sqrt{x^{\prime 2}+\left(\Delta \tau \hbar^{-1}\right)^{2}}\left[\sqrt{x^{2}+\left(\Delta \tau \hbar^{-1}\right)^{2}}+\sqrt{x^{\prime 2}+\left(\Delta \tau \hbar^{-1}\right)^{2}}\right]}
$$

where $x=\epsilon \tau \hbar^{-1}$ and $x^{\prime}=\epsilon^{\prime} \tau \hbar^{-1}$. It is verified easily by analytic calculations that the double integration of Eq. (7) results in $2 \pi$ if $\Delta \tau \hbar^{-1}=0$, giving $\chi_{\mathrm{s}} / \chi_{\mathrm{n}}=1$ as expected.

We have performed the double integration of Eq. (7) numerically by means of the trapezoidal rule dividing each region $-1000<x\left(x^{\prime}\right)<-1,-1<x\left(x^{\prime}\right)<1$, and $1<x\left(x^{\prime}\right)$
$<1000$ by 10000 meshes with equal width in each region. The result is shown in Fig. 1 in which the squares represent $\chi_{\mathrm{s}} / \chi_{\mathrm{n}}$ for $\Delta \tau \hbar^{-1}<1$ and the circles that for $\Delta \tau \hbar^{-1}>1$, respectively. It is remarked that these numerically obtained points can be fitted well by a rather simple expression as seen in Fig. 1: 


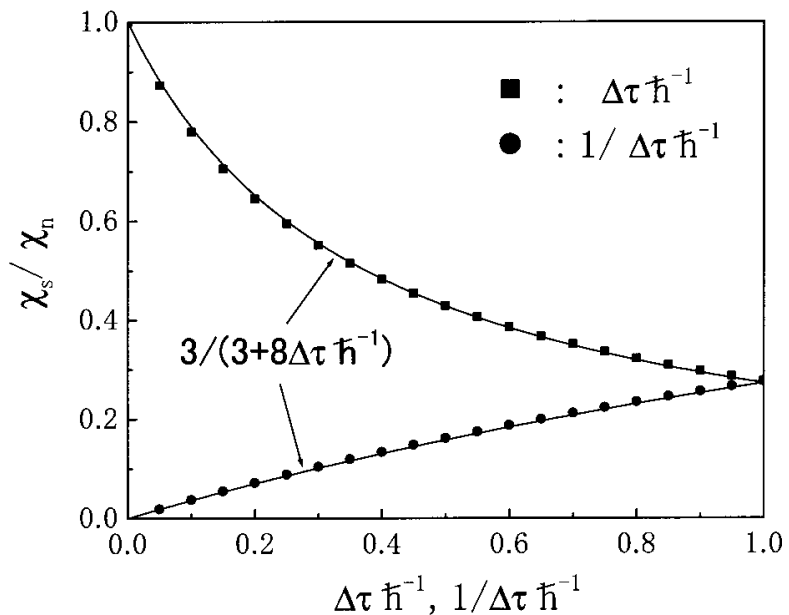

FIG. 1. Knight shift vs impurity scattering rates associated with spin-orbit coupling.

$$
\frac{\chi_{\mathrm{s}}}{\chi_{\mathrm{n}}} \approx \frac{3}{3+8 \Delta \tau \hbar^{-1}} .
$$

This behaves in the two limits $\Delta \tau \hbar^{-1} \ll 1$ and $\Delta \tau \hbar^{-1} \gg 1$ as follows:

$$
\frac{\chi_{\mathrm{s}}}{\chi_{\mathrm{n}}} \simeq \begin{cases}1-\frac{8}{3} \Delta \tau \hbar^{-1}, & \Delta \tau \hbar^{-1} \ll 1, \\ \frac{3}{8}\left(\Delta \tau \hbar^{-1}\right)^{-1}, & \Delta \tau \hbar^{-1} \gg 1 .\end{cases}
$$

The numerical coefficients in these limiting forms are considerably different from Eqs. (9) and (10) of Ref. 1 in which those are given as

$$
\frac{\chi_{\mathrm{s}}}{\chi_{\mathrm{n}}} \simeq \begin{cases}1-2 \Delta \tau \hbar^{-1}, & \Delta \tau \hbar^{-1} \ll 1, \\ \frac{1}{6}\left(\Delta \tau \hbar^{-1}\right)^{-1}, & \Delta \tau \hbar^{-1} \gg 1 .\end{cases}
$$

In conclusion, we have reanalyzed the formula of Anderson for the Knight shift of BCS superconductors subject to spinorbit scattering due to impurities, and obtained a technically exact result (8) which may give a new clue for analysis of the Knight shift experiments of BCS superconductors.

One of the authors (K.M.) acknowledges J. Flouquet and his colleagues for hospitality at CEA-Grenoble where the last stage of this work was performed. This work was supported in part by a Grant-in-Aid for COE Research (10CE2004) of Monbusho, the Ministry of Education, Science, Sports and Culture of Japan.

${ }^{1}$ P.W. Anderson, Phys. Rev. Lett. 3, 325 (1959). 